\title{
AVALIAÇÃo DA INFLUÊNCIA DOS PROCESSOS DE LECITINAÇÃO E DE AGLOMERAÇÃO NAS PROPRIEDADES FÍSICAS DE ACHOCOLATADO EM PÓ ${ }^{1}$
}

\author{
Fernanda Zaratini VISSOTTO ${ }^{2, *}$, Flávio Martins MONTENEGRO ${ }^{2}$, \\ Juliane Moreira dos SANTOS ${ }^{3}$, Sílvia Juliana Rodrigues de OLIVEIRA ${ }^{4}$
}

\begin{abstract}
RESUMO
O objetivo deste trabalho é comparar a solubilidade, a molhabilidade e a distribuição granulométrica de achocolatado em pó, submetido aos processos de: simples mistura; mistura e lecitinação $(0,3 ; 0,5 ; 0,8$ e $1 \%$ de lecitina de soja); e mistura, lecitinação $(0,3 ; 0,5 ; 0,8$ e $1 \%$ de lecitina ) e aglomeração. Ao comparar o produto obtido pelo processo de simples mistura com o lecitinado, constata-se que a presença da lecitina melhora a solubilidade do produto em todos os tratamentos utilizados. O processo de aglomeração, após a lecitinação, incrementa ainda mais a solubilidade do produto, determinada neste trabalho pelo índice de retenção - IR. Comparandose o IR dos tratamentos estudados, foram obtidos os resultados: $\mathrm{IR}_{\text {mistura }}=2,65 \% ; \mathrm{IR}_{0,3 \% \text { lec. }}=2,07 \%$; e $\mathrm{IR}_{0,3 \% \text { lec.aglom. }}=0,79 \%$. Q 3 uanto à molhabilidade, observa-se que os produtos lecitinados apresentaram menores tempos em relação aos submetidos a simples mistura, e o processo de aglomeração proporcionou uma diminuição ainda maior destes tempos $\left(\mathrm{t}_{\text {mistura }}=8 \mathrm{~min} 16 \mathrm{~s} ; \mathrm{t}_{0.3 \% \text { lec. }}=4 \mathrm{~min} 33 \mathrm{~s}\right.$; $\mathrm{t}_{0.3 \% \text { lec.aglom. }}=3 \mathrm{~min}$ ). Não se observou influência do processo de lecitinação sobre a distribuição granulométrica da bebida em pó. Já o processo de aglomeração ocasionou modificação significativa nesta propriedade.

Palavras-chave: aglomeração, lecitinação, propriedades físicas, bebidas em pó.
\end{abstract}

\section{SUMMARY}

EVALUATION OF THE INFLUENCE OF LECITHINATION AND AGGLOMERATION ON THE PHYSICAL PROPERTIES OF A COCOA POWDER BEVERAGE (COCOA POWDER BEVERAGE LECITHINATION AND AGGLOMERATION). The aim of this article is to compare the solubility, wettability and granulometric distribution of a cocoa powder beverage, which went through the following process: simple blending; blending and lecithination $(0.3 ; 0.5 ; 0.8$ and $1.0 \%$ of soy lecithin) and blending, lecithination $(0.3 ; 0.5 ; 0.8$ and $1.0 \%$ of soy lecithin) and agglomeration. Comparing the product obtained with the simple blending process and the lecithinated product, the conclusion is that the presence of lecithin improves the powder beverage solubility in all the conditions used. The agglomeration process, after lecithination, improves the solubility of the product, which was determined in this article using the retention index - RI. Comparing this parameter to the different treatments studied, the following results were obtained: $\mathrm{RI}_{\text {blend }}=2.65 \%$; $\mathrm{RI}_{0.3 \mathrm{lec}}=2.07$; $\mathrm{RI}_{0.3 \text { lec.aglom. }}=0.79 \%$. Regarding wettability, it was observed that the lecithinated products had less time if compared to the powder that went through the simple blending process, and the agglomeration process reduced even more in these times $\left(\mathrm{t}_{\text {blend }}=8\right.$ min $16 \mathrm{~s}$; $\mathrm{t}_{0,3 \% \text { lec }}=4 \mathrm{~min} 33 \mathrm{~s} ; \mathrm{t}_{0.3 \% \text { lec aglom. }}=3 \mathrm{~min}$ ). No influence of the lecithination process in the granulometric distribution was observed. The agglomeration process caused a modification in this property.

Keywords: agglomeration, lecithination, physical properties, powder beverage.

\section{1 - INTRODUÇÃO}

A importância dos alimentos em pó deve-se à sua versatilidade no manuseio, armazenamento, processo de fabricação, estabilidade química e microbiológica, entre outras. Alguns exemplos desta classe de alimentos são: leites (integral e desnatado); alimentos destinados a crianças em fase de aleitamento; bebidas à base de cacau, café e malte; café solúvel; sopas desidratadas instantâneas; suplementos protéicos; pré-misturas para panificação; leveduras; enzimas; aromas; entre outros.

${ }^{1}$ Recebido para publicação em 8/11/2005. Aceito para publicação em 6/7/2006 (001635)

${ }^{2}$ Instituto de Tecnologia de Alimentos (ITAL),

Centro de Pesquisa e Desenvolvimento de Cereais e Chocolate,

(CEREAL CHOCOTEC), Av. Brasil, 2880, Jardim Brasil,

CEP 13073-001, C. P. 139, Campinas (SP), Brasil.

E-mail:vissotto@ital.sp.gov.br.

${ }^{3}$ Engenharia de Alimentos,

Universidade Estadual do Centro-Oeste (UNICENTRO),

Guarapuava (PR)

${ }^{4}$ Engenharia de Alimentos,

Universidade Estadual de Campinas (UNICAMP), Campinas (SP)

* A quem a correspondência deve ser enviada
O processo de simples mistura dos componentes do alimento, que normalmente é empregado pelas indústrias processadoras de pós, não é garantia de que o produto apresentará uma qualidade final desejável. Têm sido utilizados, portanto, processos de lecitinação e aglomeração, os quais modificam as propriedades físicas das misturas e contribuem para que a reconstituição dos pós em meio líquido seja otimizada.

O termo "instantâneo" tem sido empregado para descrever os alimentos em pó que são facilmente miscíveis em água fria.

A função básica da lecitina de soja é a de revestimento físico das partículas, principalmente daquelas que contêm gordura em sua composição, de tal forma que, quando em meio aquoso, haja uma redução da tensão superficial entre as fases sólida e líquida.

Como o radical ácido graxo da lecitina é solúvel em óleo e o radical fosfato-colina é solúvel em água, é justificada a característica intrínseca da molécula de lipofilicidade e hidrofilicidade. Por outro lado, os fosfolipídios, pela sua tendência a formar películas ou filmes em torno das partí- 
culas sólidas ou semi-sólidas, quando entram em contato com os líquidos, evitam a formação de grumos, mantendo a dispersão estável [7, 10].

Segundo DASHIELL [1], as lecitinas podem ser aplicadas de diferentes formas visando à obtenção do efeito técnico desejado. Dentre os processos utilizados comercialmente para a aplicação da lecitina nos pós, o autor destaca a utilização de sprays em sistemas com misturadores do tipo ribbon, misturadores em V para líquido-sólidos e secadores de leito fluidizado/aglomeradores. As lecitinas podem ser aplicadas na forma pura ou, quando a tecnologia do leito fluidizado é utilizada, tanto na forma pura quanto em solução.

As principais variáveis que influenciam no processo de lecitinação são o tipo e geometria do misturador; a especificação do bico de aspersão; a pressão de ar comprimido no sistema; a vazão, a temperatura de aplicação e o tipo da lecitina, sendo o processo mais facilmente conduzido com lecitinas de baixa viscosidade, de aproximadamente 30 poise a $25^{\circ} \mathrm{C}[3]$.

Segundo MAUREL [5], o processo de aglomeração é uma técnica que permite transformar um produto ou uma mistura de produtos em pó em grânulos, modificando a sua estrutura física. Possibilita que misturas de pós-heterogêneos, na composição e tamanho das partículas, apresentem grânulos maiores e mais homogêneos, sendo as características do produto obtido dependentes dos seus constituintes, da técnica e das condições de processo utilizadas. Busca-se com o processo de aglomeração a obtenção de alimentos em pó instantâneos e a ausência de pós-finos (partículas com tamanho inferior a $50 \mu \mathrm{m}$ ), durante o processamento e manipulação.

Na reconstituição de um alimento em pó, quando as partículas finas entram em contato com o meio de dissolução, ocorre a formação de uma camada hidrófoba que impede o líquido de penetrar. A aglomeração promove a formação de uma estrutura porosa no grânulo, com aumento na quantidade de ar intersticial, formando "canais" que facilitam a entrada do líquido de dissolução [5].

A reconstituição do pó ocorre pelo umedecimento e subseqüente substituição do ar intersticial por água, por capilaridade. Se o volume de ar é pequeno, como o que ocorre nos pós não aglomerados, a quantidade de água será pequena, resultando numa solução concentrada de alta viscosidade na superfície do pó. Quando o volume de ar é grande, como o que ocorre nos pós aglomerados, a quantidade de água de dissolução será grande, facilitando assim a dispersão dos grânulos, antes da formação de soluções de alta viscosidade.

Os alimentos em pó apresentam diferentes propriedades físicas (tamanho e distribuição das partículas, densidade aparente e de partículas, porosidade, solubilidade, molhabilidade, dispersibilidade, entre outras), sendo que a medida e a caracterização destas propriedades ajudam a definir o produto, os parâmetros do processo de produção e os fenômenos que influenciam no seu comportamento [9].
Segundo PEÑA [6], quando um pó é “despejado” sobre a superfície de um líquido, as etapas que ocorrem são: molhabilidade (wettability), que consiste na penetração do líquido para o interior da estrutura do pó devido à capilaridade; imersibilidade (sinkability), que é a imersão das partículas ou de porções do pó no líquido; dispersibilidade, ou seja, a dispersão do pó no líquido; e a solubilidade, que é a dissolução das partículas pelo líquido, desde que estas sejam solúveis.

SCHUBERT [8] afirma que as propriedades associadas a essas quatro etapas são denominadas de "instantaneidade" do produto. MAIA \& GOLGHER [4] relatam em seus estudos que estas propriedades controlam os fenômenos relacionados com a reconstituição ou rehidratação do pó e que estas ocorrem de forma simultânea, o que poderia dificultar a percepção clara e imediata das distinções entre os parâmetros correspondentes.

A molhabilidade, também conhecida como umectabilidade, pode ser caracterizada pela susceptibilidade das partículas em serem penetradas pela água, estando relacionada principalmente ao conteúdo e características da gordura presentes no alimento, bem como a fatores físicos, especialmente tamanho e forma das partículas e temperatura da água de reconstituição. Produtos em pó que apresentam baixa molhabilidade tendem a formar grumos durante a adição e mistura com a água [4]. De acordo com MAIA \& GOLGHER [4], existe uma correlação inversa entre a molhabilidade e o conteúdo de gordura livre. Estudos apontaram que durante a estocagem a gordura livre permeia a partícula e uma parte atinge a superfície, tornando-a repelente à água. O espaço intersticial apresentado por partículas grandes e de forma irregular favorece a molhabilidade. Ao contrário, partículas pequenas e simétricas acarretam redução dos interstícios, prejudicando a penetração de água. É evidente que a granulometria deve ser uniforme ou com o mínimo teor de finos, os quais tendem a preencher os interstícios das partículas maiores, prejudicando a molhabilidade.

A solubilidade é um dos parâmetros utilizados para verificar a capacidade do pó para manter-se em mistura homogênea com a água, ou seja, a estabilidade da mistura [4], que freqüentemente não constitui uma solução, no sentido estrito da palavra, mas sim uma emulsão e/ou suspensão, cujo aumento da estabilidade é comumente referido como “solubilização". Segundo os mesmos autores, o termo solubilidade é inexato quando aplicado a produtos que não se constituem apenas de ingredientes solúveis, como o leite em pó, mas contêm também proteínas de dimensões coloidais e/ou gorduras. O termo foi consagrado na literatura sobre reconstituição de leite em pó, introduzindo um critério peculiar, relacionado à qualidade de hidratação.

A maioria das técnicas propostas para determinação da solubilidade pode ser enquadrada em uma das duas classes gerais, apontadas por MAIA \& GOLGHER [4], as que estimam a quantidade de material que é dissolvido e as que determinam a quantidade de material insolúvel. De modo geral, os testes incluídos na segunda categoria são mais sensíveis e envolvem as etapas de mistura e homoge- 
neização de quantidades conhecidas da amostra e da água, em temperatura controlada; centrifugação ou repouso da "solução" sob condições prefixadas; separação do sobrenadante, secagem e pesagem do resíduo.

São poucos os estudos que constam da literatura científica sobre os processos de lecitinação e aglomeração de alimentos em pó, o que torna necessária à realização de pesquisas que possibilitem um melhor entendimento destes processos, seja separados e/ou combinados, e de sua influência nas propriedades físicas dos pós. A escolha de um achocolatado em pó para o estudo dos processos deveu-se, principalmente, à presença do componente hidrófobo na sua composição, a manteiga de cacau.

A proposta deste artigo científico é a de apresentar uma comparação entre algumas propriedades físicas (solubilidade, molhabilidade e distribuição granulométrica) de um achocolatado, submetido a simples mistura de seus constituintes, lecitinação e lecitinação seguida de aglomeração.

\section{2 - MATERIAL E MÉTODOS}

\section{1 - Material}

No estudo, foi utilizado um achocolatado em pó constituído de 50,70\% de sacarose de cana (açúcar cristal), $20,00 \%$ de cacau em pó alcalino, 20,00\% de leite desnatado, $9,00 \%$ de maltodextrina (17-19,9 D.E.) e 0,30\% de aroma de baunilha.

A lecitina de soja utilizada foi a natural de baixa viscosidade ( $\mathrm{T}=24{ }^{\circ} \mathrm{C} / 29$ poise $)$, denominação comercial Lecsam L, fornecida pela Solae Company.

\section{2 - Métodos}

\subsection{1 - Mistura}

Os componentes básicos (sacarose, cacau, leite, maltodextrina e aroma de baunilha) foram misturados em misturador ribbon, com capacidade para $20 \mathrm{~L}$ de pó. A mistura foi realizada à temperatura ambiente $\left( \pm 25^{\circ} \mathrm{C}\right)$, por $20 \mathrm{~min}$ a $120 \mathrm{rpm}$.

\subsection{2 - Lecitinação}

Após a mistura, no mesmo equipamento, realizou-se a lecitinação dos pós, sendo mantidas as mesmas condições de temperatura e rotação das pás. Os teores de lecitina utilizados foram de 0,$3 ; 0,5 ; 0,8$; e $1,0 \%$ e, pela determinação da massa de lecitina a ser incorporada no produto, determinou-se o tempo de aspersão.

O sistema de aspersão da lecitina sobre a mistura de pós era constituído de um tanque pulmão pressurizado (2,0 bar), com capacidade para 1,5 L de lecitina, acoplado a um bico aspersor da Spraying Systems (especificação: $1 / 4 \mathrm{~J}, 73320 \mathrm{SS}$ ). O bico aspersor estava posicionado no centro da parte superior do misturador ribbon (conforme especificado na Figura 1).

Na Figura 1, tem-se uma representação esquemática do misturador e do sistema de aspersão da lecitina nos produtos em pó.

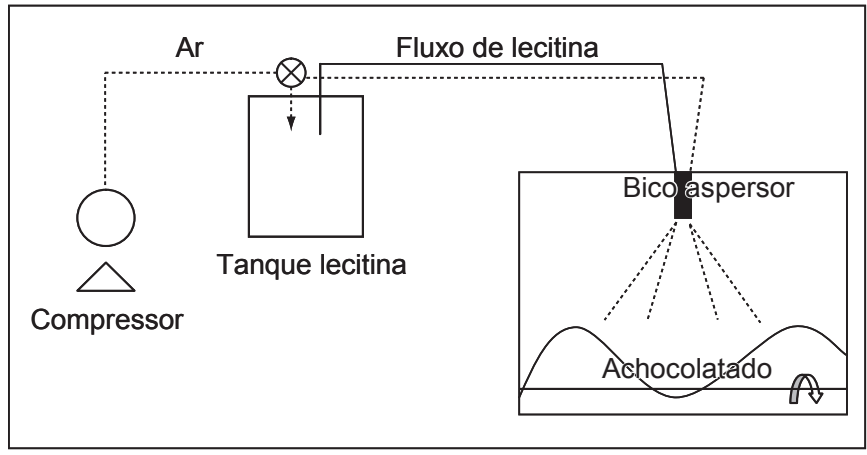

FIGURA 1 - Equipamento de mistura e sistema de aspersão utilizado na etapa de lecitinação.

\subsection{3 - Aglomeração}

Utilizou-se nesta etapa um aglomerador piloto, marca I.C.F Industrie Cibec (Figura 2), sendo a alimentação do equipamento realizada através de uma tremonha, no interior da qual havia uma escova rotativa que forçava a passagem do pó contra uma grelha de abertura quadrada de $1 \mathrm{~mm}$.

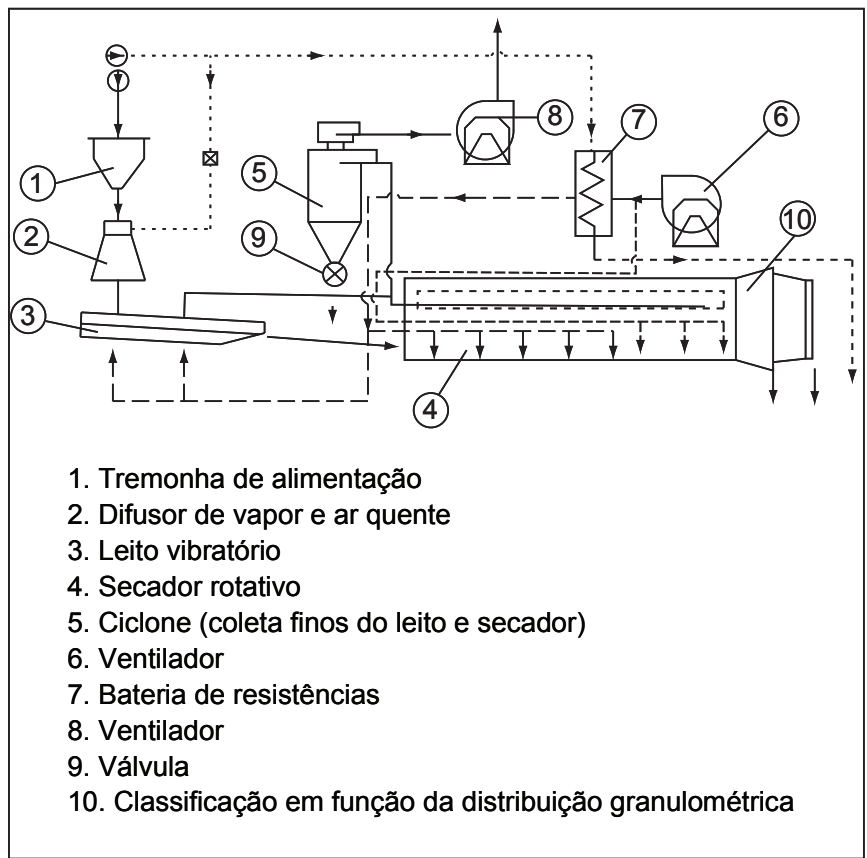

FIGURA 2 - Desenho esquemático do aglomerador (I. C. F Industrie Cibec s. p. a.)

A aglomeração ocorreu pelo contato direto do pó, que encontrava-se à temperatura ambiente $\left(25^{\circ} \mathrm{C}\right)$, com o vapor saturado (2) (pressão: 1,5 bar), havendo a condensação do vapor na superfície do produto na forma de microgotículas e posterior formação de uma solução concentrada e viscosa. As partículas, cujas superfícies eram colantes, uniam-se 
umas às outras e se aglomeravam, formando grânulos, que em um leito vibratório (3) recebiam um fluxo de ar quente no sentido ascendente, tendo início o processo de secagem. Os grânulos formados caiam em seguida em um secador rotativo (4) (temperatura: $100^{\circ} \mathrm{C}$ ), sendo que, após a secagem, os grânulos eram selecionados de acordo com a granulometria obtida.

\subsection{4 - Propriedades físicas}

Determinou-se a solubilidade, a molhabilidade e a distribuição granulométrica das amostras que foram submetidas aos processos de simples mistura; mistura e lecitinação; e mistura, lecitinação e aglomeração.

\section{3 - Solubilidade}

A análise consistiu em adicionar 20,0 g da amostra em $150 \mathrm{~mL}$ de água destilada a $25^{\circ} \mathrm{C}$, em béquer de $600 \mathrm{~mL}$. Em seguida, realizou-se a mistura utilizando agitador mecânico (300 rpm, 20 s) e uma "hélice" desenvolvida especialmente para esta análise, de forma a proporcionar uma mistura mais efetiva do pó com o líquido. A "hélice" desenvolvida era de chapa de aço inoxidável com 0,2 mm de espessura, sendo que a parte que entrava em contato com o líquido era vazada, de formato quadrado, e com duas chapas unindo as diagonais. A solução era filtrada em peneira com abertura de 32 mesh e, quantificada a solução passante pela peneira (líquido mais sólidos solúveis). A solubilidade foi medida pelo índice de retenção (IR), que é a relação entre a massa de insolúveis e solúveis. Os resultados apresentados são a média de 3 determinações.

$I R \%=\frac{100\left(170-m_{\text {soluçăo passante peneira }}\right)}{m_{\text {soluçăo passante peneira }}}$

\section{4 - Molhabilidade}

Esta propriedade foi medida adaptando-se metodologia proposta por HLA [2]. Um equipamento em acrílico (Figura 3) foi utilizado nesta análise. O método consistia na queda de
2,0 g de amostra sobre $400 \mathrm{~mL}$ de água destilada a $25^{\circ} \mathrm{C}$, em béquer de $600 \mathrm{~mL}$, e medição do tempo necessário para que todas as partículas molhassem, determinado visualmente. As determinações foram realizadas em triplicata.

\section{5 - Distribuição granulométrica}

Para este teste, foram utilizadas peneiras com diferentes aberturas (850, 710, 600, 500, 425, 350 e 300 micra) mais o fundo. A quantidade de amostra utilizada foi de $100 \mathrm{~g}$, e as peneiras com a amostra foram levadas ao equipamento Granutest, com o reostato na posição 10 , por um período de 5 min. Na quantificação da massa retida nas peneiras, foi utilizada uma balança semi-analítica com precisão de $\pm 0,01$ g. Os resultados apresentados são a média de três determinações.

\section{3 - RESULTADOS E DISCUSSÃo}

\section{1 - Solubilidade}

Na Figura 4, são apresentados os resultados da análise de solubilidade das bebidas em pó submetidas aos diferentes processos. A solubilidade foi determinada de forma indireta, pelo IR, sendo que a maior solubilidade do produto está associada com menores valores de IR. As amostras obtidas apenas pela simples mistura apresentaram IR de $2,65 \%$, superior aos das outras amostras submetidas aos processos de lecitinação e lecitinação seguida por aglomeração. Pode-se afirmar, portanto, que o processo de lecitinação incrementou a solubilidade dos achocolatados, assim como o processo de aglomeração, sendo este ainda mais eficiente. O processo de lecitinação foi efetivo na melhora da solubilidade do pó ao se utilizar teor de lecitina igual a 0,5\%. A utilização de quantidades superiores a esta não contribuiu para o incremento na solubilidade do produto. A lecitina foi efetiva no produto aglomerado até $0,3 \%$, quantidades superiores não resultaram em melhora significativa na solubilidade da bebida em pó estudada.
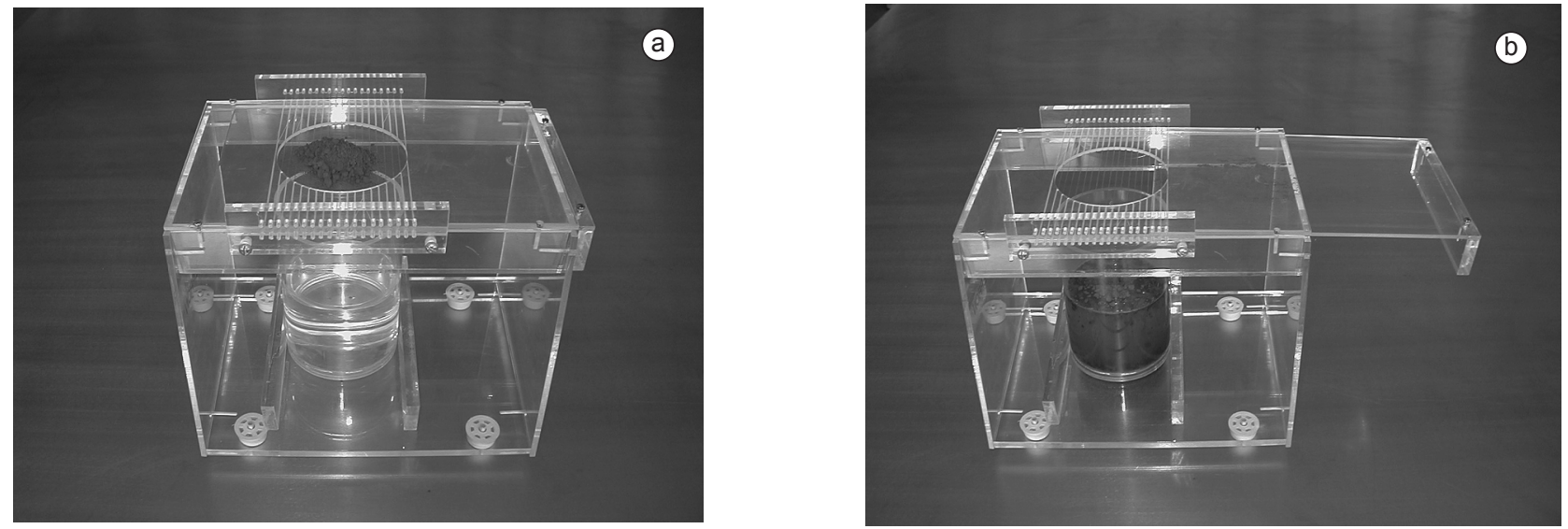

FIGURA 3 - Equipamento para avaliação de molhabilidade. a) 2 g de amostra sobre o alçapão; e b) Análise em curso. 


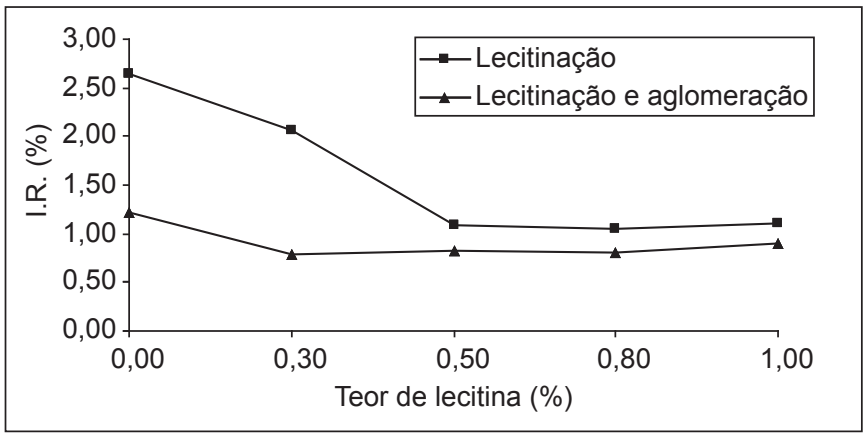

FIGURA 4 - Solubilidade da bebida em pó à base de cacau submetida aos processos de lecitinação e lecitinação e aglomeração.

\section{2 - Molhabilidade}

Os resultados da análise de molhabilidade dos pós submetidos aos diferentes processos são apresentados no Figura 5. Verifica-se que a presença de lecitina é bastante favorável a esta propriedade. Os pós lecitinados apresentaram menores tempos para que as partículas se molhassem em relação aos que foram submetidos ao processo de simples mistura, melhora que pode ser observada até um teor máximo de $0,5 \%$ de lecitina, sendo que teores superiores a este não melhoraram significativamente a molhabilidade das partículas de pó. Isso se explica devido à propriedade "molhante" e "emulsionante" que a lecitina exerce sobre o produto. Com relação ao processo de aglomeração, percebe-se que um tempo ainda menor é necessário para que as partículas se molhem, sendo a condição ótima o produto previamente lecitinado com $0,3 \%$. Nesta condição, a bebida em pó apresenta-se completamente instantânea. Acima deste teor de lecitina, não se observa melhora significativa da molhabilidade do produto.

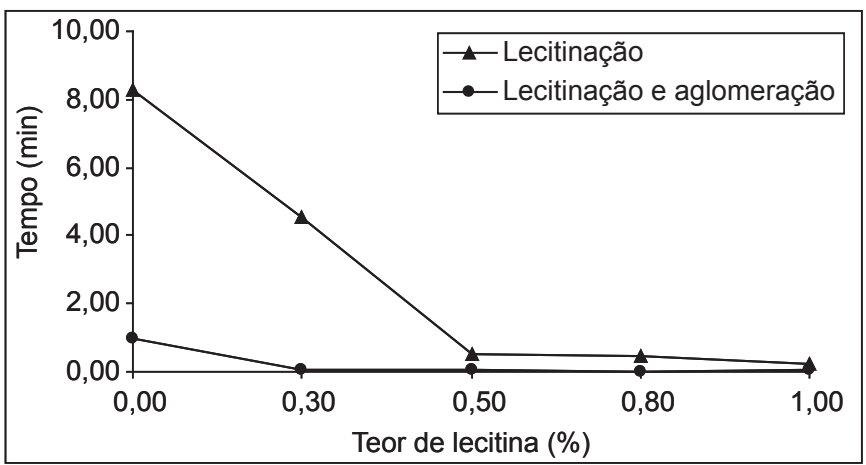

FIGURA 5 - Molhabilidade da bebida em pó à base de cacau submetida aos processos de lecitinação e lecitinação e aglomeração.

\section{3 - Distribuição granulométrica}

As Figuras 6 e 7 apresentam os resultados das análises de distribuição granulométrica para os achocolatados com diferentes teores de lecitina, antes e após o processo de aglomeração.

Analisando-se a Figura 6, observa-se que a lecitina não influencia na granulometria do achocolatado, visto que praticamente não houve diferença nas porcentagens de pó retido em uma mesma peneira para os diferentes teores de lecitina utilizados.

É possível verificar que a aglomeração ocasiona uma modificação na distribuição granulométrica do produto com relação ao produto apenas lecitinado, conforme apresentado nas Figuras 6 e 7, sendo uma maior quantidade de massa retida nas peneiras de maior abertura (850 - 500 micra).

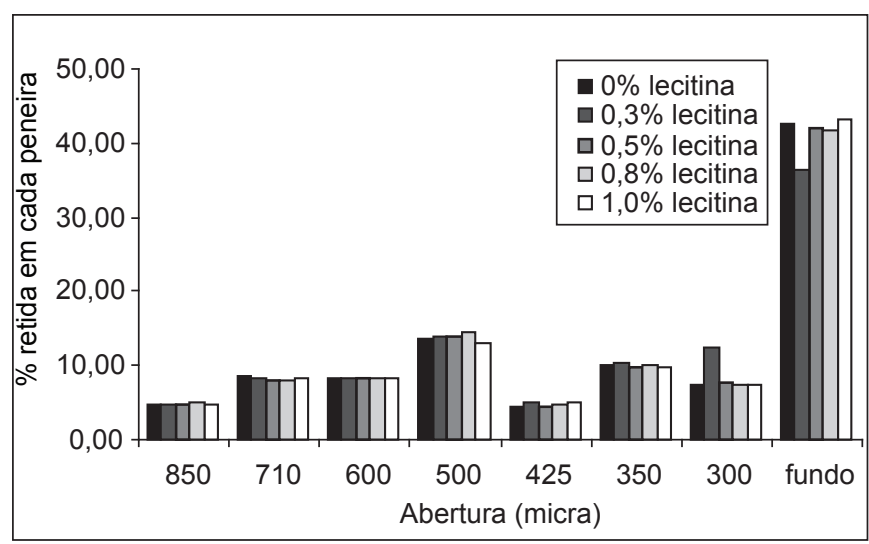

FIGURA 6 - Distribuição granulométrica da bebida em pó à base de cacau submetida ao processo de lecitinação.

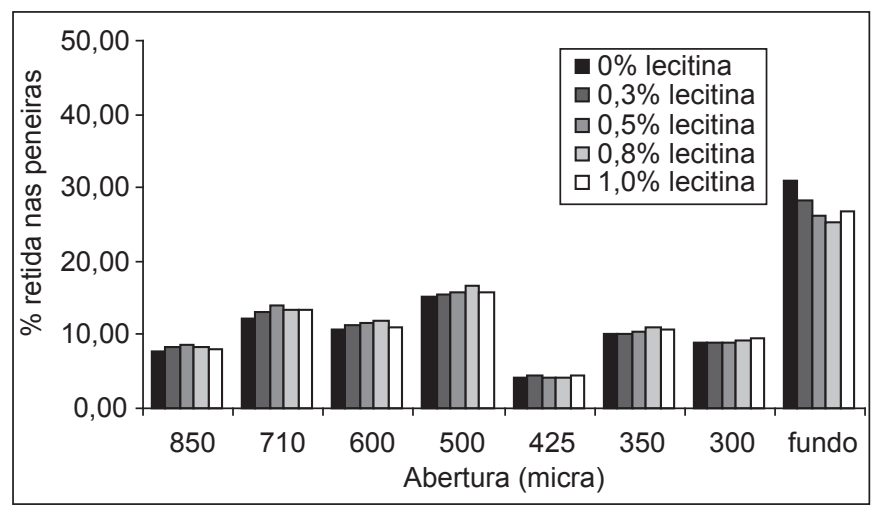

FIGURA 7 - Distribuição granulométrica da bebida em pó à base de cacau submetida aos processos de lecitinação e aglomeração.

O processo de aglomeração levou a um aumento do diâmetro médio de partícula para todos os teores de lecitina testados, conforme apresentado na Figura 8. Os maiores aumentos no diâmetro médio de partícula foram obtidos para as formulações de achocolatados com teores de lecitina de 0,$5 ; 0,8 ; 1,0$ e $0,3 \%$, respectivamente.

\section{4 - CONCLUSÕES}

Conclui-se que a presença de lecitina melhora a solubilidade e a molhabilidade do produto estudado e o processo de aglomeração, após a lecitinação, melhora ainda mais estas propriedades físicas. No processo de lecitinação, a condição ótima obtida foi de $0,5 \%$ de lecitina e no processo de lecitinação, seguido de aglomeração, a condição ótima foi de $0,3 \%$ de lecitina. O processo de aglomeração altera a 


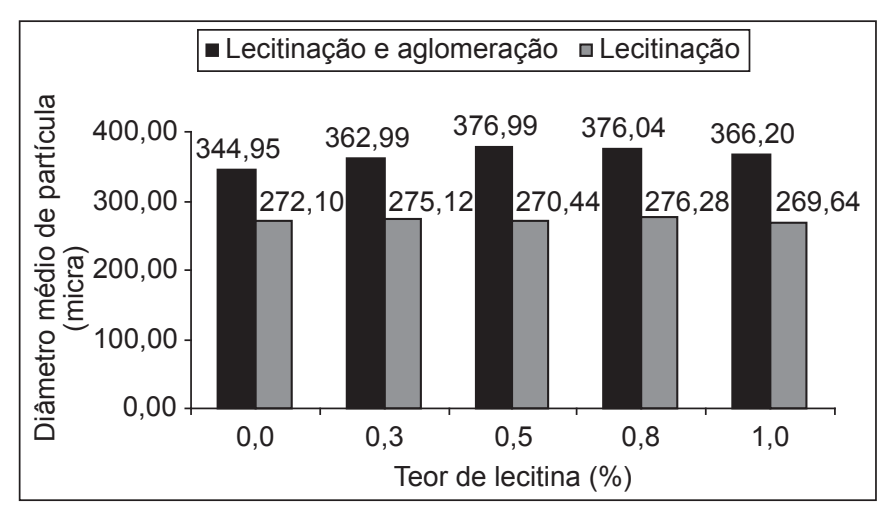

FIGURA 8 - Comparação entre os diâmetros médios de partículas para os processos de lecitinação e lecitinação e aglomeração para achocolatado em pó.

distribuição granulométrica da bebida em pó e aumenta o diâmetro médio de partícula em todos os teores de lecitina estudados.

\section{5 - REFERÊNCIAS BIBLIOGRÁFICAS}

[1] DASHIElL, G. L. Lecithin in Food Processing Applications. In: SZUHAJ, Lecithins, Sources Manufacture \& Uses, Illinois, American Oil Chemists Society, p. 213-224. 1989

[2] HLA, P. K.; HOGEKAMP, S. Wetting behaviour of instanized cocoa beverage powders. International Journal of Food Science and Technology, v. 34, n. 4, p. 335-342, 1999.

[3] MACHADO, R. P. Tecnologia de lecitinação de produtos em pó. In: VISSOTTO, F. Z.; LUCCAS, V. Alimentos em pó formulados com cacau, café e derivados lácteos.
Manual Técnico Seminário organizado Cereal Chocotec/Ital, Campinas, SP, 2002, p. 29-47.

[4] MAIA, A. B. R.; GOLGHER, M. Parâmetros para Avaliação da Qualidade de Reconstituição do Leite em pó Desidratado em Secador de Aspersão (“Spray-Drier”). Boletim SBCTA, Campinas, v. 17, n. 3, p. 235-254. julho/setembro, 1983.

[5] MAUREL, E.C. Tecnologie des poudre. Granulation: tout un savior-faire. Process, n ${ }^{\circ}$ 1095, p. 62-65, 1994.

[6] PEÑA, L. M. R., Estudo da instantaneização de misturas contendo cacau em pó por aglomeração com jato de vapor, Campinas, 2003, 259p. (Tese de dissertação de doutorado) - Faculdade de Engenharia de Alimentos, Universidade Estadual de Campinas.

[7] SALAZAR, J. Aspectos gerais sobre a produção de leite em pó instantâneo. Revista do Instituto de Laticínios “Cândido Tostes", v. 34, n. 204, p. 15-25, julho/agosto, 1979.

[8] SCHUBERT, H. Food particle technology. Part I: properties of particles and particles food systems. Journal of food engineering, v. 6, n. 1, p. 1-30, 1993.

[9] TEUNOU, E., FitZPATRICK, J. J., SYNOTT, E. C. Characterisation of food powder flowability. Journal of Food Engineering, v. 39, n. 1, p. 31-37, janeiro, 1999.

[10] TURATTI, J. M. Lecitina: obtenção e uso. Boletim ITAL, Campinas, v. 17, n. 3, p. 235-251, julho/setembro, 1980.

\section{6 - AGRADECIMENTOS}

Os autores agradecem ao CNPq a concessão de bolsa de iniciação científica, que possibilitou o levantamento de parte dos dados experimentais do estudo. 